\title{
Modified serial transverse small-bowel enteroplasty procedure: A novel salvage technique for lengthening the gastric conduit for esophageal reconstruction
}

\author{
George Rakovich, MD, ${ }^{\mathrm{a}}$ and Nelson Piché, MD, ${ }^{\mathrm{b}}$ Montréal, Québec, Canada
}

\footnotetext{
From the a Division of Thoracic Surgery, Department of Surgery, Hôpital Maisonneuve-Rosemont, University of Montreal, Montréal, Québec, Canada; and ${ }^{\mathrm{b}}$ Division of Pediatric Surgery, Department of Surgery, Centre Hospitalier Universitaire Sainte-Justine, University of Montreal, Montréal, Québec, Canada.

This work did not receive any funding.

Disclosures: Dr Rakovich receives Personal Fees from Medtronic. Dr Piché has nothing to disclose with regard to commercial support.

Received for publication Jan 11, 2019; revisions received Feb 22, 2019; accepted for publication March 2, 2019; available ahead of print April 13, 2019

Address for reprints: George Rakovich, MD, Department of Surgery, Hôpital Maisonneuve-Rosemont, 5415 Boulevard de l'Assomption, Montréal, Québec, Canada H1T 2M4 (E-mail: george.rakovich@umontreal.ca). J Thorac Cardiovasc Surg 2019;158:e77-9

$0022-5223 / \$ 36.00$

Copyright (c) 2019 by The American Association for Thoracic Surgery

https://doi.org/10.1016/j.jtcvs.2019.03.011
}

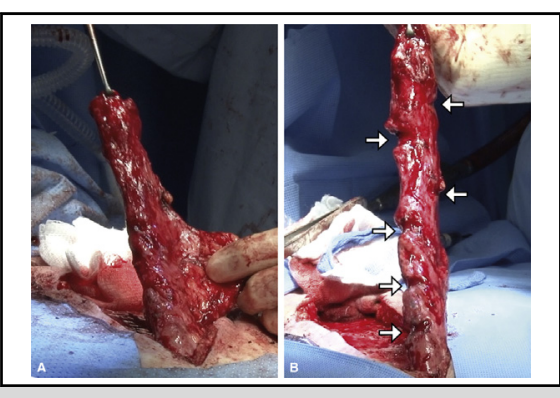

Myotomies achieving lengthening of gastric conduit for esophageal reconstruction.

Central Message

A series of transverse myotomies can increase the length of a gastric conduit sufficiently to bridge a 3-cm gap and allow primary gastroesophageal reconstruction after esophagectomy.
Video clip is available online.

Insufficient gastric conduit length can be a major problem when undertaking esophageal reconstruction. In pediatric surgery, serial transverse small-bowel enteroplasty (STEP) involves a series of partial transverse transections of a congenitally obstructed, dilated loop of bowel, transforming bowel width into length. We were confronted with a case in which gastric tip necrosis after gastroesophageal reconstruction in the neck resulted in a $2.5-\mathrm{cm}$ long tissue deficit at the anastomosis. Basing our technique on the principle underlying STEP, we performed a series of transverse myotomies along the gastric conduit that achieved a sufficient gain in length to allow an adequately perfused, tension-free anastomosis.

\section{CASE PRESENTATION}

A 70-year-old patient with a T3N0 adenocarcinoma of the cardia underwent a minimally invasive 3-field esophagectomy. On postoperative day 7 , imaging showed a tear in the membranous trachea in addition to an anastomotic leak. The anastomosis was taken down and replaced anteriorly to avoid the risk of an aerodigestive fistula while the tracheal tear was repaired.

Because the retrosternal and left pleural spaces were not available as a result of previous coronary artery bypass grafting, the conduit was routed subcutaneously, and a new end-to-side esophagogastrostomy was performed in the neck. While the patient recovered from multiple physiologic complications, this anastomosis broke down, and at 8 weeks, there was a loss of gastric substance and a tissue deficit of $2.5 \mathrm{~cm}$ in length.

Relative to the stomach, jejunal and colonic conduits are technically difficult and prone to ischemia, and functional results are variable. With the objective of achieving primary gastroesophageal reconstruction, we therefore considered the STEP technique used in pediatric small-bowel atresia. STEP accomplishes lengthening through a series of partial transverse transections on alternating sides of a dilated loop of bowel. ${ }^{1}$ The bowel can then be unraveled like an accordion, transforming width into length (Figure 1). The gastric conduit was 4-cm wide along its length, which was considered be sufficient to allow transverse divisions without causing undue narrowing. We had 2 concerns with this approach: Firstly, the imperative of maintaining the integrity of the gastroepiploic pedicle was believed to limit any potential gains to be made along the mesenteric border of the greater curvature. Secondly, the disruption of the submucosal plexus resulting from transections was believed to carry a potential risk of conduit ischemia. Therefore, after mobilization of the subcutaneous conduit, the integrity of stomach perfusion was assessed intraoperatively after the application of bulldog clamps at the planned transection sites. On visual inspection, clamping was found to interrupt 
A
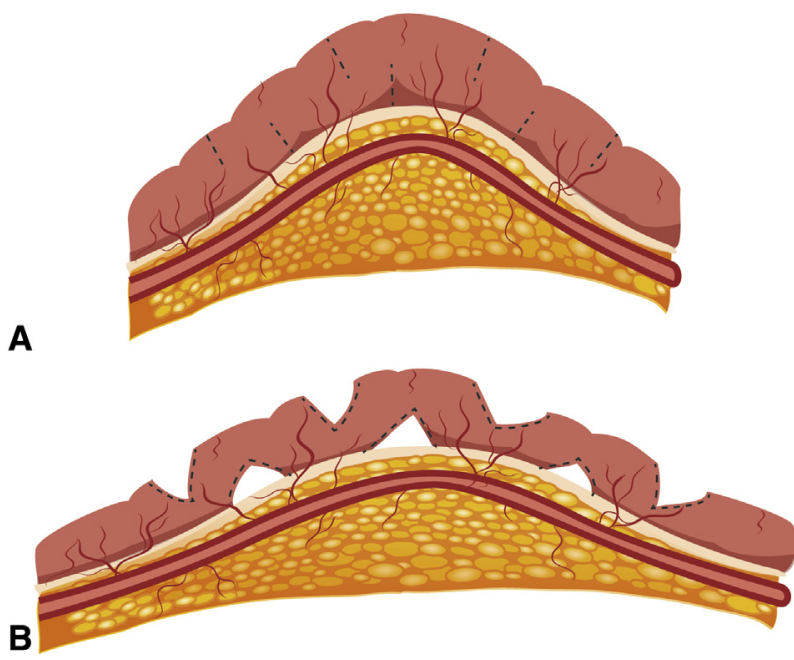

FIGURE 1. Illustration of transverse small-bowel enteroplasty in small bowel atresia: a series of partial transverse transections at the locations indicated by dotted lines on alternating sides of a dilated loop of bowel (A) transform width into length (B).

arteriolar blood flow to the gastric tip, which was restored on clamp removal (tonometry and fluorescent dye angiography were not readily available at our institution at the time).
Myotomies were therefore substituted for full-thickness transection to preserve the submucosal plexus. Myotomies were carried across the staple line (carefully preserving the mucosa), while the gastric wall in proximity to the gastroepiploic pedicle was left undisturbed and perfusion at the tip was continually reassessed (Figure 2). A series of 6 myotomies achieved a gain of $3 \mathrm{~cm}$ in conduit length, sufficient to allow an adequately perfused, tension-free anastomosis (Video 1).

There was a delayed anastomotic leak 2 weeks postoperatively. Endoscopy documented good perfusion of the gastric conduit, with damage to the esophageal remnant sustained during a difficult dissection that contributed to the leak. This healed with a stenosis that was definitively resolved with temporary stenting. Four years after the procedure, the patient has no symptoms of delayed gastric emptying, and there is no food retention on follow-up endoscopies.

\section{DISCUSSION}

Insufficient gastric conduit length can be a major problem when undertaking esophageal reconstruction in the neck. $^{2,3}$ Obtaining adequate conduit length depends foremost on complete mobilization of the stomach and duodenum. $^{2,3}$ Unconventional techniques to obtain
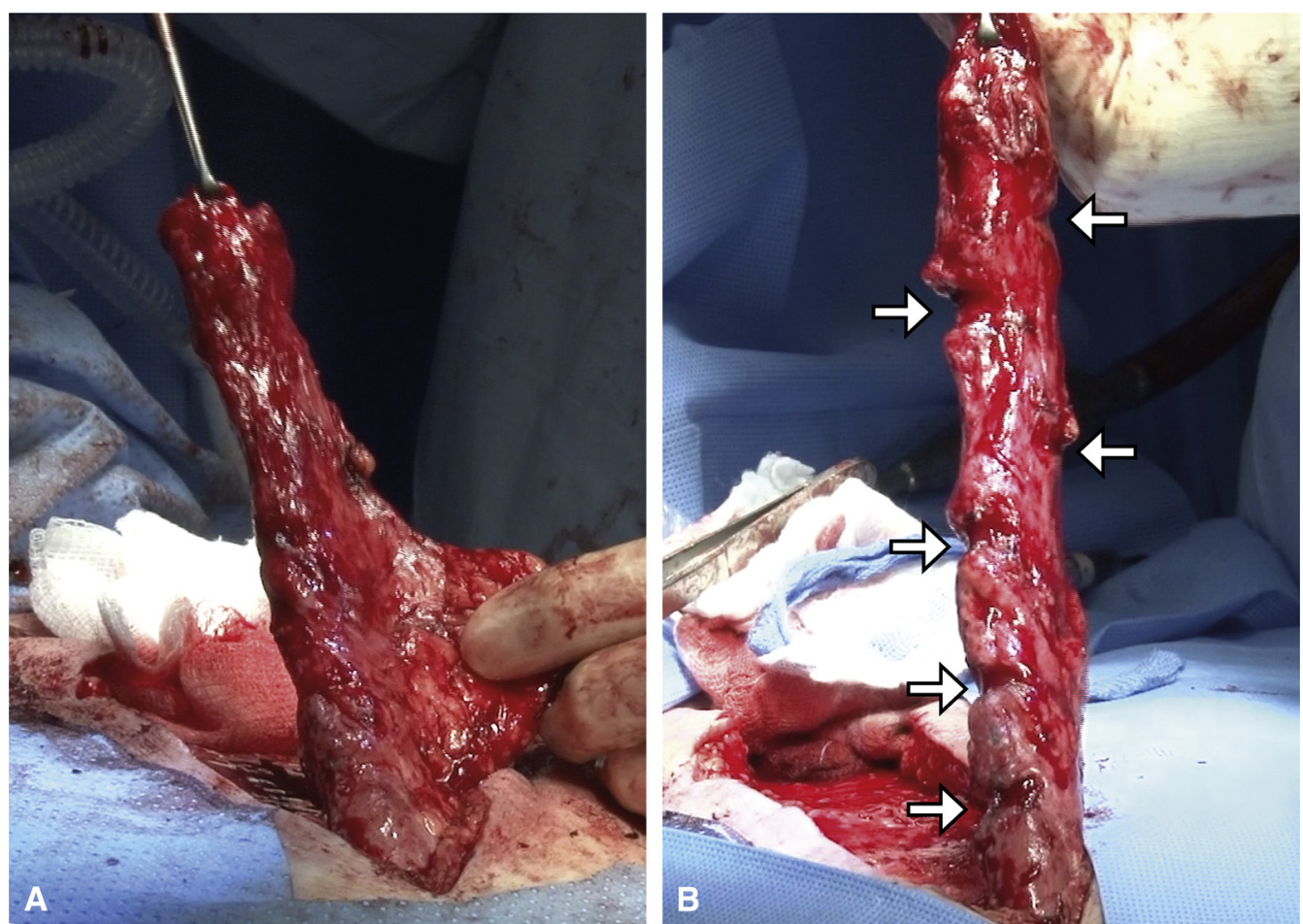

FIGURE 2. A, Two months after esophagectomy, a gastric conduit routed subcutaneously has been mobilized. B, A series of transverse myotomies (marked by arrows) along the gastric conduit achieved a gain in length of $3 \mathrm{~cm}$, allowing bridging of a gap with the cervical esophagus resulting from gastric tip necrosis and performance of a well-perfused, tension-free anastomosis. 


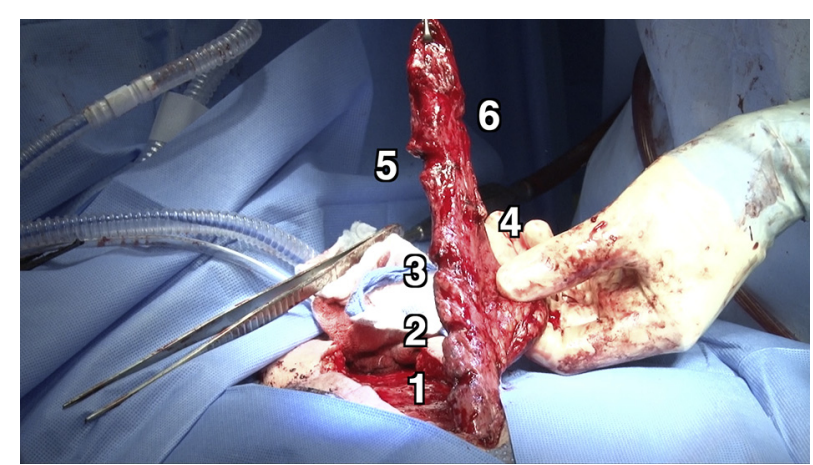

VIDEO 1. Two months after esophagectomy, a gastric conduit routed subcutaneously is mobilized. A series of transverse myotomies along the gastric conduit are performed and achieve a gain in length of $3 \mathrm{~cm}$, allowing bridging of a gap with the cervical esophagus that had resulted from gastric tip necrosis. A well-perfused, tension free anastomosis can now be performed. Video available at: https://www.jtcvs.org/article/S0022-5223(19) 30679-8/fulltext.

additional length include a procedure described by Matsubara and colleagues ${ }^{3}$ that is conceptually similar to STEP in that it also involves partial transverse transection of the gastric tube; however, transection is limited to a single firing of the stapler across the antrum at the level of the incisura. Kitayama and associates ${ }^{2}$ described a variation of the technique of Matsubara and colleagues ${ }^{3}$ in which a transverse myotomy with longitudinal closure of the seromuscular layer is substituted for full-thickness transection, with the objective of preserving the submucosal vascular plexus, which is critical for maintaining adequate perfusion of the gastric conduit. These techniques may result in a gain of 4 to $5 \mathrm{~cm}$ in length. 2,3

Other, more complex techniques include duodenal transection to release the gastric conduit while keeping the right gastroepiploic pedicle intact ${ }^{4}$ and fundus rotation gastroplasty, ${ }^{5}$ which requires an intact fundus and a "supercharging" vascular anastomosis in the neck.

The advantages of our technique are that it was fairly straightforward to perform and that it allowed us to achieve our objective of primary reconstruction in an expedient way. The drawbacks are that the gain in length was modest and that the applicability in an immediate setting in which the gastric vascular network has not yet adapted is unclear. The main lengthening effect was likely due to the release of the muscularis along the staple line. Additional myotomies along the greater curvature may have had only a modest effect and should be weighed against the potential risk of injuring the gastroepiploic pedicle.

\section{References}

1. Sudan D, Thompson J, Botha J, Grant W, Antonson D, Raynor S, et al. Comparison of intestinal lengthening procedures for patients with short bowel syndrome. Ann Surg. 2007;246:593-601; discussion 601-4.

2. Kitayama J, Kaisaki S, Ishigami H, Hidemura A, Nagawa H. Angleplasty in gastric tube reconstruction after esophagectomy. Dis Esophagus. 2009;22:418-21.

3. Matsubara T, Ueda M, Uchida C, Takahashi T. Modified stomach roll for safer reconstruction after subtotal esophagectomy. J Surg Oncol. 2000;75:214-6.

4. Yoshida N, Baba Y, Oda E, Kosumi K, Ishimoto T, Watanabe M, et al. Reconstruction using a pedunculated gastric tube with duodenal transection after esophagectomy and pharyngolaryngectomy. Ann Surg Oncol. 2015;22:4352.

5. Uhl W, Strobel O, Friess H, Schilling M, Büchler MW. Fundus rotation gastroplasty: rationale, technique and results. Dis Esophagus. 2002;15:101-5. 